\title{
Learning Dynamic Bayesian Belief Networks Using Conditional Phase-Type Distributions
}

\author{
Adele Marshall ${ }^{1}$, Sally McClean ${ }^{1}$, Mary Shapcott ${ }^{1}$, and Peter Millard ${ }^{2}$ \\ ${ }^{1}$ School of Information and Software Engineering, University of Ulster, Jordanstown. \\ BT37 0QB, Northern Ireland. UK \\ \{AH.Marshall, SI.McClean, CM.Shapcott\}@ulst.ac.uk \\ ${ }^{2}$ Department of Geriatric Medicine, St George's Hospital, London. SW17 0QT, UK \\ P.Millardesghms.ac.uk
}

\begin{abstract}
In this paper, we introduce the Dynamic Bayesian Belief Network (DBBN) and show how it can be used in data mining. DBBNs generalise the concept of Bayesian Belief Networks (BBNs) to include a time dimension. We may thus represent a stochastic (or probabilistic) process along with causal information. The approach combines BBNs for modelling causal information with a latent Markov model for dealing with temporal (survival) events. It is assumed that the model includes both qualitative (causal) and quantitative (survival) variables. We introduce the idea of conditional phase-type (C-Ph) distributions to model such data. These models describe duration until an event occurs in terms of a process consisting of a sequence of phases - the states of a latent Markov model. Our approach is illustrated using data on hospital spells (the process) of geriatric patients along with personal details, admissions reasons, dependency levels and destination (the causal network).
\end{abstract}

\section{Introduction}

Bayesian Belief Networks (BBNs) are statistical graphical models which encapsulate causal information and provide a framework for describing and evaluating probabilities using a network of inter-related variables. BBNs have been discussed by a number of authors. [1] has described the use of such models as providing a "unified qualitative and quantitative way for representing and reasoning with probabilities and independencies". Dependency may result from some sort of causal mechanism coming into play. Such causality is often represented by means of a BBN [2]. Benefits of using such a representation include the incorporation of prior knowledge, the provision of validation and insight, and the ability to learn causal interactions [3]. Such an approach may lead to the development of probabilistic expert systems [4].

Dynamic Bayesian Belief Networks (DBBNs) generalise BBNs to include a time dimension. We may thus represent a stochastic (or probabilistic) process along with causal information [5] [6]. There have been various efforts to extend the standard theory of BBNs to allow time to be modelled. [7] provide a temporal definition ofcausal dependence where a set of variables indexed by time is associated with each cause and with an effect. Other approaches have been introduced by [8-13]. 
In statistical theory, Markov models are often used to represent stochastic processes. Structured phase-type $(\mathrm{Ph})$ distributions [14] describe duration until an event occurs in terms of a process consisting of a sequence of latent phases. For example, duration of stay in hospital can be thought of as a series of transitions through phases such as: acute illness, intervention, recovery or discharge. This captures how a domain expert conceptualises the process. We can prove that any such statistical distribution may be represented arbitrarily closely by one of phase-type form [15].

\section{The Model}

Our aim is to provide an approach which uses probabilistic temporal reasoning to describe a process which commences with an initiating event and may terminate in a number of different ways. The initiating event may be described in terms of a BBN. We begin by presenting a motivating example, as follows.

\subsection{A Motivating Example:}

Consider a situation where, at time zero, an elderly person has a traumatic event, such as a stroke or a fall, and is admitted to hospital where she remains until discharge at time t. Discharge may be either due to death or discharge alive to one of a number of destinations such as "own home" or "nursing home". On admission to hospital, data are available on a number of variables which may be regarded as causal with respect to the traumatic event e.g. domestic situation, or previous state of health. The ability to predict this length of stay in hospital is important both to the geriatrician and to the hospital manager.

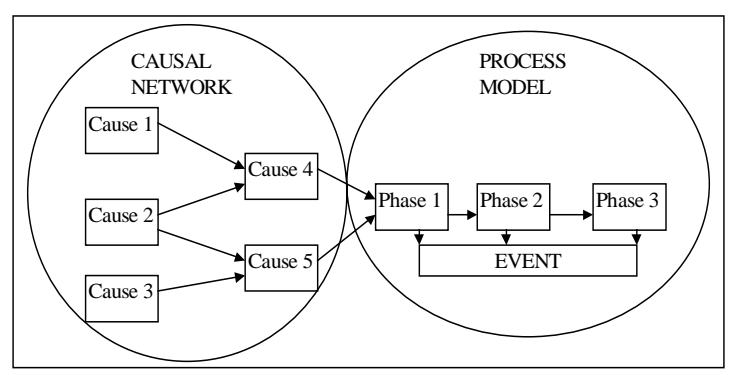

Fig. 1. The underlying representation describes a DBBN in terms of a number of interrelated causal nodes which temporally precede and predetermine (in a probabilistic sense) the effect node(s) which constitute the process. The effect node(s) here are characterised by a continuous positive random variable(s), the duration, described by a phase-type distribution

We introduce a model which combines a BBN (causal network) with a process model (Fig. 1.). The Process Model may be defined in a manner similar to that of [5][16] where we consider an event $\langle\mathrm{E}\rangle$ which initiates a process $\mathrm{P}$ at time zero and $\langle\mathrm{P}, \mathrm{t}\rangle$ indicates that the process $\mathrm{P}$ is active at time $\mathrm{t}$. Then prob $\langle\mathrm{P}, \mathrm{t}\rangle$ is the probability that the process is still active at time t. $\operatorname{Prob}<\mathrm{P}, \mathrm{t}>$ is known as the survivor 
function, denoted by $\mathrm{F}(\mathrm{t})$ and, for a continuous time representation, its derivative $\mathrm{f}(\mathrm{t})$ is the probability density function (p.d.f.) of the time for which the process is active. Here we define $\mathrm{f}(\mathrm{t})$ by

$\mathrm{f}(\mathrm{t}) \mathcal{d}=\operatorname{prob}($ process terminates in $(\mathrm{t}, \mathrm{t}+\mathscr{d}) \mid$ process is still active at $\mathrm{t}$ ).

Some previous work has been done on data of this sort, (including both qualitative quantitative variables) mainly involving the introduction of conditional Gaussian (CG) distributions [17][18]. We here introduce the idea of Conditional Phase-type (C$\mathrm{Ph}$ ) distributions which are more appropriate for process data.

\section{Learning DBBNs Using Conditional Phase-Type Distributions}

\subsection{Phase-Type Distributions}

To describe the probability $\operatorname{prob}<\mathrm{P}, \mathrm{t}>$ that the process is still active at time $\mathrm{t}$ we will use a sub-class of phase-type distributions, the Coxian sub-class of phase-type distributions [19]. These have the latent states (or phases) ordered with the process starting in the first of these and then developing by either sequential transitions through these phases or transitions into the absorbing state (the terminating event, phase $n+1$ ). Such phases may then be used to describe stages of a process which terminates at some stage. For example, in the context of durations of hospital treatment, patients may be thought of as passing through (latent) stages of diagnosis, assessment, rehabilitation and long-stay care where most patients are eventually rehabilitated and discharged. We define a phase-type distribution, as follows: Let $\{X(t) ; t \geq 0\}$ be a (latent) Markov chain in continuous time with states $\{1,2, \ldots n, n+$ 1 ) with $X(0)=1$, and

$$
\operatorname{prob}\{X(t+\delta t)=i+1 \mid X(t)=i\}=\lambda_{i} \delta t+o(\delta t)
$$

and for $i=1,2, \ldots n$

$$
\operatorname{prob}\{X(t+\delta t)=n+1 \mid X(t)=i\}=\mu_{i} \delta t+o(\delta t) .
$$

Here states $\{1,2, \ldots n\}$ are latent (transient) states of the process and state $n+1$ is the (absorbing) state initiated by the terminating event, $\lambda_{1}$ represents the transition from state $\mathrm{i}$ to state $(\mathrm{i}+1)$ and $\mu_{\mathrm{i}}$ the transition from state $\mathrm{i}$ to the absorbing state $(n+1)$. [20] developed the theory of Markov chains such as those defined by (1) and (2). The probability density function of $T$ is as follows;

$$
f(\mathrm{t})=\mathbf{p} \exp \{\mathbf{Q} \mathrm{t}\} \mathbf{q}
$$

where:

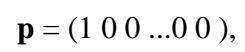

$$
\begin{aligned}
& \mathbf{q}=-\mathbf{Q} \mathbf{1}=\left(\mu_{1} \mu_{2} \ldots \mu_{\mathrm{n}}\right)^{\mathrm{T}} .
\end{aligned}
$$


and the matrix $\mathbf{Q}$ is:

$$
\mathbf{Q}=\left[\begin{array}{cccccc}
-\left(\lambda_{1}+\mu_{1}\right) & \lambda_{1} & 0 & \ldots & 0 & 0 \\
0 & -\left(\lambda_{2}+\mu_{2}\right) & \lambda_{2} & \ldots & 0 & 0 \\
: & : & : & \ldots & : & : \\
0 & 0 & 0 & \ldots & -\left(\lambda_{\mathrm{n}-1}+\mu_{\mathrm{n}-1}\right) & \lambda_{\mathrm{n}-1} \\
0 & 0 & 0 & \ldots & & -\mu_{\mathrm{n}}
\end{array}\right],
$$

with the $\lambda_{i}$ 's and $\mu_{\mathrm{i}}$ 's from (1) and (2) (Fig. 2). Equation (3) gives the Coxian phase-type distribution (p.d.f.), with the $\lambda_{1}$ 's describing transitions through the ordered transient states $\{1,2, \ldots n\}$ and the $\mu_{\mathrm{i}}$ 's transitions into the (single) absorbing state $n+1$.

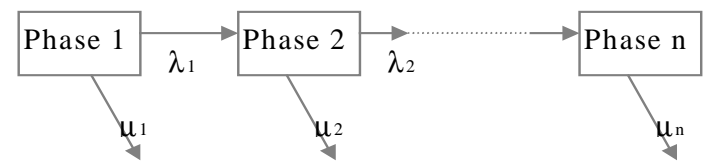

Fig. 2. An illustration of phase-type distributions where $\lambda_{1}$ represents the transition from phase $i$ to phase $(\mathrm{i}+1)$ and $\mu_{\mathrm{i}}$ the transition from phase $\mathrm{i}$ to the absorbing phase $(n+1)$

\subsection{The Dynamic Bayesian Belief Network (DBBN)}

In the DBBN we have causal nodes $\mathbf{C}=\left\{\mathrm{C}_{1}, \ldots \mathrm{C}_{\mathrm{m}}\right\}$ and process nodes $\mathbf{P h}=\left\{\mathrm{Ph}_{1}, \ldots \mathrm{Ph}_{\mathrm{n}}\right\}$ where the causal nodes comprise a BBN in the usual sense. We may therefore represent the joint distribution of $\mathbf{C}$ by:

$$
\mathrm{P}(\mathbf{C})=\prod_{\mathrm{i}} \mathrm{P}\left(\mathrm{C}_{\mathrm{i}} \mid \mathrm{pa}\left(\mathrm{C}_{\mathrm{i}}\right)\right)
$$

where pa is the parent set of $\mathrm{C}_{\mathrm{i}}$. The distribution of the process nodes $\mathbf{P h}$ is then given by the p.d.f.:

$$
f(\mathrm{t} \mid \mathrm{pa}(\text { process }))=\mathbf{p} \exp \{\mathbf{Q} \mathrm{t}\} \mathbf{q}
$$

where pa(process) are the causal nodes which are parents of the process and $\mathbf{p}, \mathbf{Q}$ and $\mathbf{q}$ vary according to the values of the causal nodes in pa(process). The process may therefore be considered locally independent of the causal network.

When the topology of the causal network is known a priori we may induce the DBBN by using maximum likelihood methods to fit parameters to phase-type distributions for each set of values in pa(process). A sequential procedure (unsupervised learning) is adopted whereby increasing numbers of $\mathrm{n}$ phases are tried, starting with $\mathrm{n}=1$ (corresponding to the exponential distribution), until no improvement in the fit to the data can be obtained by adding a new phase. Such an approach may be implemented by using a series of likelihood ratio tests [21]. If the causal network topology is not known a priori, likelihoods for different models may be compared until the optimal topology is found. 


\subsection{Learning Incomplete DBBNs}

It is frequently the case that DBBNs represent data which is incomplete. Duration of stay is typically missing since learning of processes is often incremental and for some individuals the length of stay will not be known when data collection ceases; typically the cessation of data collection occurs at the current time point. Such data are known in the statistical literature as right censored and are a common feature of survival data of which our process is an example. More generally, such data are referred to as being incomplete. For our model such incompleteness may be straightforwardly taken account of by incorporating appropriate terms into the likelihood functions. The likelihoods may then be maximised using the EM (Expectation-Maximisation) algorithm [21][22]. Use of the EM algorithm with phase-type distributions is described by [23].

\section{An Application to Geriatric Medicine}

The data analysed in this example relate to geriatric patients at St George's Hospital, London. Durations of hospital stay (the process) were available for patients along with personal details, admission reasons, patient dependency levels and outcome (the causal network). In total there were 4730 patients details recorded in the clinical computer system (Clinics) between 1994-1997. The significance of each individual Clinics variable on patient length of stay was tested using Chi-square tests, the most significance of these are included in Fig. 3.

These variables have been used as input into CoCo [24 a computer package capable of learning BBNs. The package uses the maximum likelihood approach to obtain the causal networks which best describe the data set. It does so by comparing the complete model with all possible candidate models. Fig. 3 is one of various candidate models that can represent the causal relations between the Clinics variables.

The BBN consists of a number of interrelated causal nodes which are grouped into blocks according to the time domain to which they relate. The causal network in Fig. 3 comprises of four such blocks. Whenever a patient is admitted to a geriatric ward, their personal details are recorded (block 1 - personal details), they then undergo an initial medical examination to investigate their condition or illness (block 2 admission reasons). Following the medical examination, the patient is assessed for level of dependency to carry out everyday activities. The score given to patients is known as the Barthel score which is categorised into grades and used in the third block in the BBN. After spending a period of time in hospital (duration of stay) the patient will experience one of three events; discharge from hospital, transfer to another ward or hospital or death (destination). This is the terminating event, the last node in the BBN. The network is further described in [25].

Fig. 3 is an illustration of how the Clinics variables might be interrelated. In consultation with the geriatricians and the Chi-square test results of significance, we selected three of the most significant of these variables to produce a simplified model similar in form to that in Fig. 1. The nodes (gender and stroke) represent the causal network while duration of stay, the continuous variable defines the process model. 


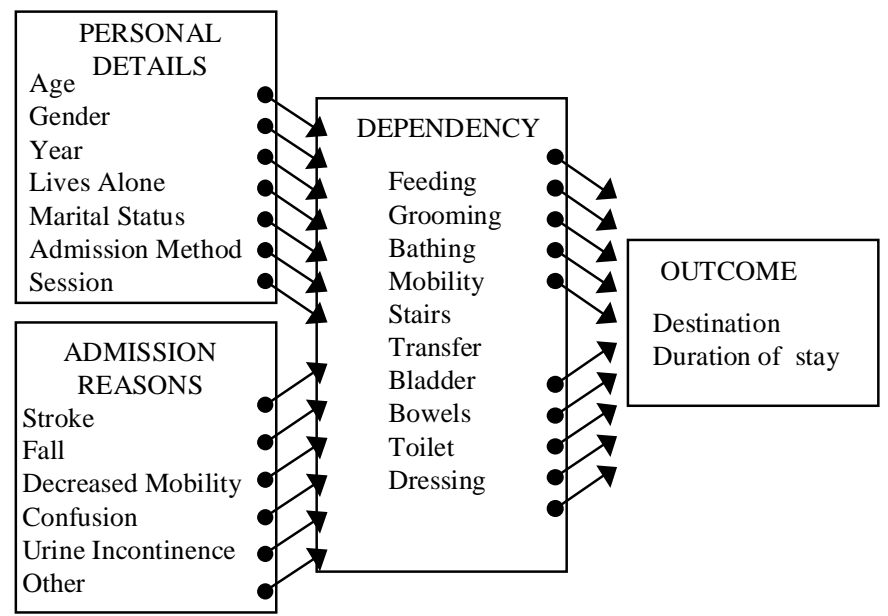

Fig. 3. An illustration of how the Clinics variables might be interrelated.

Similar data to the Clinics data set is described in 26 where a two phase distribution is fitted and reported to be a reasonable approximation to the data. We have here fitted a general phase-type distribution to the continuous variable, duration of stay. We induced the network displayed in Fig. 4.

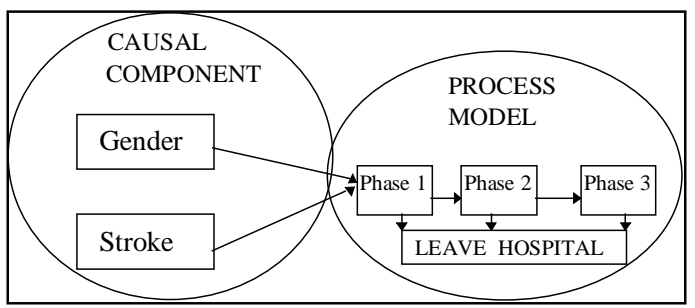

Fig. 4. DBBN for the Clinics data set where there are two causal nodes, gender and stroke, which temporally precede the process variable, 'duration of stay'

The number of phases in each of the cohorts of patient length of stay was induced by unsupervised learning. The p.d.f. of the Coxian phase-type distribution is represented by equation (7). The parameters from (1) and (2), which describe this distribution, are determined using maximum likelihood function:

$$
L=\prod_{\mathrm{g}=1}^{2} \prod_{\mathrm{s}=1}^{2}\left(\mathrm{p}_{\mathrm{gs}}\right)^{\mathrm{n}_{\mathrm{gs}}} \prod_{\mathrm{i}} f_{\mathrm{gs}}\left(\mathrm{t}_{\mathrm{i}}\right)
$$

where $g$ represents the gender, $s$ the stroke variable, $n_{g s}$ the number of patients in cohort gs and $f_{\mathrm{gs}}$ the corresponding p.d.f. Each cohort of patients is separately fitted to a phase-type distribution. The sum of these represents the log-likelihood function (Table 1). MATLAB software [27] is used to implement the Nelder-Mead algorithm [28] to perform the likelihood ratio tests which determine the optimal number of phases in the distribution. The estimates for the $\mu_{i}$ and $\lambda_{1}$ parameters of the phase-type distributions are displayed in Table 2 The optimality was also assessed using the least 
squares approach; an alternative to the likelihood ratio test. Both approaches yielded similar results.

Table 1. Phase-Type Distributions - displays the results of fitting phase-type distributions to the data. The * indicates the optimal number of phases for each particular patient cohort.

\begin{tabular}{ll}
\hline Patient Cohort & Log-Likelihood \\
\hline Male Stroke & $\mathrm{n}=1, \mathrm{~L}=-481.87 ; \mathrm{n}=2, \mathrm{~L}=-452.77^{*} ; \mathrm{n}=3, \mathrm{~L}=-452.46$ \\
Male Non-Stroke & $\mathrm{n}=1, \mathrm{~L}=-7828.3 ; \mathrm{n}=2, \mathrm{~L}=-6415.1^{*} ; \mathrm{n}=3, \mathrm{~L}=-6414.1$ \\
Female Stroke & $\mathrm{n}=1, \mathrm{~L}=-775.96 ; \mathrm{n}=2, \mathrm{~L}=-705.49^{*}$ \\
Female Non-Stroke & $\mathrm{n}=1, \mathrm{~L}=-17251 ; \mathrm{n}=2, \mathrm{~L}=-13725 ; \mathrm{n}=3, \mathrm{~L}=-13705 ; \mathrm{n}=4, \mathrm{~L}=-13700^{*}$ \\
\hline
\end{tabular}

Table 2. Estimates for the C-Ph Distribution

\begin{tabular}{ll}
\hline Patient Cohort & Estimates of Parameters \\
\hline Male Stroke & $\mu_{1}=0.0272 \mu_{2}=0.0012 \lambda_{1}=0.0008$. \\
Male Non-Stroke & $\mu_{1}=0.0043 \mu_{2}=0.0007 \lambda_{1}=0.0020$. \\
Female Stroke & $\mu_{1}=0.0256 \mu_{2}=0.0009 \lambda_{1}=0.0011$. \\
Female Non-Stroke & $\mu_{1}=0.0389 \mu_{2}=0.0005 \mu_{3}=0.0011 \mu_{4}=0.0001 \lambda_{1}=0.0013 \lambda_{2}=0.0009 \lambda_{3}=0.0003$. \\
\hline
\end{tabular}

\section{Summary and Conclusion}

There is extensive literature on graphical models and BBNs. However the extension of such models to dynamic situations, where time is an important factor, is an important research area which requires further attention. We believe that our approach has much to contribute in the development of this important research area.

In this paper our concern has been with providing a new technique for mining DBBNs. Our approach combines ideas from statistics for the analysis of survival data with extensive literature on BBNs. We have thus provided a methodology for probabilistic temporal reasoning. We have introduced a particular type of latent Markov model - the conditional Phase-type distribution - to describe local dependencies in relation to a stochastic process. Our resulting Dynamic Bayesian Belief Network is hybrid, in that we use discrete variables for the causal model and a continuous variable for the stochastic process. We use the EM algorithm to learn from data which are statistically incomplete and which include latent variables. A general approach to such problems has been introduced and illustrated by a simple example.

\section{References}

1. Buntine, W.: A Guide to the Literature on Learning Probabilistic Networks from Data. IEEE Transactions on Knowledge and Data Engineering, Vol. 8(2) (1996) 195-210

2. Pearl, J.: Causal Diagrams for Empirical Research. Biometrika 82 (1995) 669-710

3. Friedman, N., Goldszmidt, M., Heckerman, D., Russell, S.: Challenge: What is the Impact of Bayesian Networks on Learning? Proc. 15 $5^{\text {th }}$ Int. Joint Conf. on AI (1997) 10-15

4. Spiegelhalter, D., Cowell, R.G. Learning in Probabilistic Expert Systems. In Bernando, J.M., Berger, J.M., Dawid, A.P., Smith, A.F.M. (eds.) Bayesian Statistics 4. Oxford University Press (1992) 447-465

5. Dean, Y., Kanazawa, K.: A Model for Reasoning about Persistence and Causation. Computational Intelligence, Vol. 5(3) (1989) 142-150 
6. Russell, S., Binder, J., Koller, D., Kanazawa, K.: Local Learning in Probabilistic Networks with Hidden Variables. Proc. 14th Int. Joint Conf. on AI (1995) 1146-1152

7. Heckerman, D., Breese, J.S.: A New Look at Causal Independence. Proc. $10^{\text {th }}$ Conf. UAI (1997)

8. Aliferis, C.F., Cooper, G.F.: A Structurally and Temporally Extended BBN Model: Definitions, Properties and Modeling Techniques. Proc $12^{\text {th }}$ Conf. On UAI (1996) 28-39

9. Arroyo-Figuerca, G., Sucar, L.E., Villavicencio, A.: Probabilistic Temporal Reasoning and its Application to Fossil Power Plant Operation. Expert Sys. with Apps. 15 (1998) 317-324

10. Berzuini, C., Bellazzi, R., Quaglini, S.: Temporal Reasoning with Probabilities, Proc. of Workshop on UAI (1989)14-21

11. Kjaerulff, A.: A Computational Scheme for Reasoning in Dynamic Probabilistic Networks. Proc. $8^{\text {th }}$ Conf. On UAI (1992) 121-129

12. Nicholson, A.E., Brady, J. M.: Dynamic Belief Networks for Discrete Monitoring. IEEE Trans. on Systems, Man and Cybernetics 34(11) (1994) 1593-1610

13. Santos E.(Jnr), Young, J.D.: Probabilistic Temporal Networks: A Unified Framework for Reasoning with Time and Uncertainty. Int. J. Approx. Reasoning, 20 (1999) 263-291

14. Neuts, M.: Structured Stochastic Matrices of M/G/1 Type and Their Application. Marcel Dekker, NY (1989)

15. Faddy, M.: Examples of Fitting Structured Phase-Type Distributions, Applied Stochastic Models and Data Analysis 10 (1994) 247-255

16. Hanks, S., Madigan, D., Gavrin, J.: Probabilistic Temporal Reasoning with Endogenous Change. Proc. $11^{\text {th }}$ Conf. on UAI (1995)

17. Lauritzen, S.L., Wermuth, N.: Graphical Models For Associations Between Variables, Some of Which are Qualitative and Some Quantitative. Annals of Statistics 17 (1989) 3157

18. Gammerman, A., Luo, Z., Aitken, C.G.G., Brewer, M.J.: Computational Systems for Mixed Graphical Models. Adaptive Computing \& Information Processing. UNICOM, (1994)

19. Cox, D. Miller, H.D.: The Theory of Stochastic Processes, Methuen, London (1965)

20. Faddy, M., McClean, S.: Analysing Data on Lengths of Stay of Hospital Patients Using Phase-Type Distributions. Applied Stochastic Models and Data Analysis (2000) to appear

21. Dempster, A.P., Laird, N.M., Rubin, D.B.: Maximum Likelihood from Incomplete Data via the EM Algorithm. Journal of the Royal Statistical Society B, 39 (1977) 1-38

22. Lauritzen, S.L.: The EM Algorithm For Graphical Association Models With Missing Data. Computational Statistics and Data Analysis 19 (1995) 191-201

23. Aalen, O.O.: Phase Type distributions in Survival Analysis. Scand. J. Stat. 4 (1995) 447463

24. Badsberg, J.H.: A Guide to CoCo - An Environment for Graphical Models. Inst. of Elect. Sys., Dept. of Maths. \& Computer Science, Aalborg University, Denmark (1992)

25. Marshall, A., McClean, S., Shapcott, M., Hastie, I., Millard, P.: Developing a BBN for the Management of Geriatric Hospital Care. J. Health Care Mgt. Sc. (2000) to appear

26. McClean, S.I., Millard, P.H.: Patterns of Length of Stay After Admission in Geriatric Medicine - An Event History Approach. The Statistician 42 (1993) 263-274

27. MATLAB. Reference Guide, The MathsWorks Inc., Natick, Massachusetts (1992)

28. Bunday, B.D.: Basic Optimisation Methods. Edward Arnold Publishers Ltd, London (1984) 